\title{
Papel da Dosagem Seriada de Troponina nos Pacientes com Suspeita de Contusão Miocárdica após Trauma Torácico Fechado*
}

\author{
The Role of Serial Measurement of Troponin in Patients \\ with a Suspected Myocardial Injury after Chest Trauma
}

Thiago Domingos Corrêa ${ }^{1}$, Rogério da Hora Passos ${ }^{1}$, Danilo Teixeira Noritomi², Evandro José de Almeida Figueiredo ${ }^{3}$, Antonio Capone Neto ${ }^{2}$

\section{RESUMO}

JUSTIFICATIVA E OBJETIVOS: A contusão miocárdica está freqüentemente associada ao trauma torácico fechado. Seu correto diagnóstico é um constante desafio aos profissionais que trabalham em unidades de emergência, devido aos seus sintomas inespecíficos e a ausência de exames subsidiários com precisão para fazer o diagnóstico. Dentre os diversos métodos diagnósticos estudados, tem-se destacado nos últimos anos o papel dos indicadores de necrose miocárdica troponina I e troponina T. Por serem proteínas constituintes do aparelho de regulação contrátil celular, são liberadas na corrente sanguínea somente após a perda da integridade de membrana dos miócitos e, portanto, são altamente específicas para detectar lesão miocárdica.

1. Médico Residente da Unidade de Terapia Intensiva Adultos - Hospital Israelita Albert Einstein.

2. Médico Assistente da Unidade de Terapia Intensiva Adultos - Hospital Israelita Albert Einstein.

3. Médico Preceptor da Residência Médica da Unidade de Terapia Intensiva Adultos - Hospital Israelita Albert Einstein.

${ }^{*}$ Recebido da Unidade de Terapia Intensiva do Hospital Israelita Albert Einstein, São Paulo, SP

Apresentado em 21 de dezembro de 2006

Aceito para publicação em 01 de junho de 2007

Endereço para correspondência:

Dr. Thiago Domingos Corrêa

Unidade de Terapia Intensiva Adultos - Hospital Israelita Albert Einstein

Av. Albert Einstein, 627, Bloco A - $5^{\circ} \mathrm{A}$ - Morumbi

05651-901 São Paulo, SP

Fone: (11) 3747-1500 / Fax: (11) 3742-2834

E-mail: thiagodc@einstein.br

(C)Associação de Medicina Intensiva Brasileira, 2007
CONTEUDO: Foi realizada uma revisão de estudos clínicos nas bases eletrônicas de dados MedLine e LILACS, no período de janeiro de 1980 a novembro de 2006, sobre a importância da dosagem seriada de troponina como instrumento diagnóstico e preditor de evolução clínica desfavorável nos pacientes com contusão miocárdica.

CONCLUSÕES: Embora exista maior especificidade das troponinas I e $\mathrm{T}$ quando comparadas aos indicadores tradicionais, CKMB massa e CPK total, esses dois indicadores apresentarem sensibilidade e valor preditivo positivo baixos para diagnosticar contusão miocárdica. Pacientes que apresentam alterações eletrocardiográficas, elevação de troponinas, ou ambas, devem permanecer em observação em unidade de terapia intensiva (UTI), por no mínimo 24 horas, período em que se desenvolve a maioria das complicações decorrentes da contusão miocárdica.

Unitermos: contusão miocárdica, trauma torácico, troponina I, troponina $T$

\section{SUMMARY}

BACKGROUND AND OBJECTIVES: Myocardial contusion is often associated with blunt chest trauma. Its diagnosis is challenging to the professionals who work in emergency department due to nonspecific symptoms and the lack of auxiliary exams with enough accuracy to diagnose. Among the available diagnostic tools, the biomarkers of myocardial injury troponin I and troponin T have stood out. Troponins are proteins of the citocellular apparatus, released into the bloodstream only after the disruption of myocytes cellular membrane. Therefore they are highly specific to detect myocardial injuries. 
CONTENTS: We performed a clinical review using the electronic databases MedLine and LILACS from January 1980 to November 2006 about the importance of a serial measurement of troponin I and $T$ as a diagnostic tool as well as predictor of unfavorable clinical outcome in patients with myocardial contusion after a blunt chest trauma.

CONCLUSIONS: Although troponins I and T are more specific than usual biomarkers CKMB and CK, these two first biomarkers show a low sensitivity and positive predictive value to diagnosis myocardial contusion. Patients with ECG abnormalities, troponins elevations or both should remain in an intensive care unit (ICU) for at least 24 hours, period in which they cam develop most of the complications related to myocardial contusion.

Key Words: blunt trauma, myocardial contusion, troponin I, troponin T

\section{INTRODUÇÃO}

Sabe-se que o número de mortes devido a acidentes automobilísticos é de grande impacto nos grandes centros urbanos. Aproximadamente $16 \%$ das internações nos hospitais do sistema único de saúde (SUS) são destinados a este tipo de lesão'. Publicações científicas recentes vêm chamando cada vez mais a atenção da comunidade médica para a contusão miocárdica, devido à sua morbidade e mortalidade associadas, além da potencial gravidade dos casos não diagnosticados corretamente ${ }^{2-4}$.

A incidência de contusão miocárdica em pacientes vítimas de trauma torácico fechado varia de 3\% a 56\% dos casos, dependendo dos critérios diagnósticos utilizados $^{4}$. Seu diagnostico continua sendo um desafio aos profissionais que atendem os pacientes, vítimas de traumatismo torácico fechado. Tal fato deve-se a inexistência de sinais e sintomas específicos para esse tipo de lesão, ao amplo espectro da apresentação clínica de acordo com a sua gravidade e, principalmente, à falta de métodos subsidiários precisos para detectar a lesão miocárdica ${ }^{4}$.

Assim sendo, diversos estudos têm sido realizados nos últimos anos em busca de ferramentas que possam auxiliar no diagnóstico dessa entidade clínica ${ }^{5-10}$. Grande parte desses estudos diz respeito ao papel dos indicadores de lesão miocárdica troponina T e troponina I (Tabela 1). Estes indicadores têm sido bastante investigados já que os indicadores clássicos de lesão, como CK, CKMB (atividade e massa) e mioglobina se mostraram de pouco valor diagnóstico da contusão miocárdica ${ }^{5-10}$.

O objetivo deste estudo foi discutir a importância da dosagem seriada de troponina como instrumento diagnóstico e preditor de evolução clínica desfavorável nos pacientes com contusão miocárdica, através de levantamento bibliográfico realizado nas bases eletrônicas de dados MedLine e LILACS, no período de janeiro de 1980 a novembro de 2006 . Foram utilizados os seguintes unitermos: myocardial contusion, troponin I, troponin $T$, blunt trauma, myocardial injurie, blunt chest trauma.

\section{ILUSTRAÇÃO DE CASO CLÍNICO}

Paciente do sexo masculino, 48 anos, admitido na unidade de emergência por politraumatismo após acidente automobilístico. $O$ eletrocardiograma revelava ritmo cardíaco sinusal com freqüência cardíaca de 85 bpm, com estrassístoles ventriculares isoladas.

A evolução dos indicadores de necrose miocárdica após trauma desse paciente evidenciou importante liberação de troponina I, CKMB massa e CPK total (Tabela 2). Foi realizado ecocardiograma que não revelou alterações estruturais e da função ventricular. Foi submetido a tomografia computadorizada de tórax, que evidenciou fraturas do $5^{\circ}$ e $6^{\circ}$ arcos costais à direita e tênues opacidades em vidro fosco subjacentes a essas fraturas, compatíveis com contusão pulmonar.

Tabela 1 - Principais Estudos Prospectivos Avaliando a Precisão das Troponinas I e T no Diagnóstico da Contusão Miocárdica após Trauma Torácico Fechado.

\begin{tabular}{|c|c|c|c|c|c|c|c|c|}
\hline Autores & Anos & Pacientes & Indicadores & Valores de Corte $(\mu \mathrm{g} / \mathrm{L})$ & $\mathrm{S}(\%)$ & E (\%) & VPP (\%) & VPN (\%) \\
\hline Ferjani e col. $^{7}$ & 1997 & 128 & $\mathrm{TT}$ & $\geq 0,5$ & 31 & 91 & 16 & 82 \\
\hline Bertinchant e col. ${ }^{5}$ & 2000 & 94 & TT & $\geq 0,1$ & 12 & 100 & 100 & 74 \\
\hline Bertinchant e col. $^{5}$ & 2000 & 94 & $\mathrm{TI}$ & $\geq 0,1$ & 23 & 97 & 75 & 76.7 \\
\hline Velmahos e col. ${ }^{10}$ & 2003 & 333 & $\mathrm{TI}$ & $\geq 1,5$ & 73 & 60 & 21 & 94 \\
\hline Velmahos e col. ${ }^{10}$ & 2003 & 333 & $\mathrm{TI}+\mathrm{ECG}^{*}$ & $\geq 1,5$ & 100 & 71 & 34 & 100 \\
\hline Edouard e col. ${ }^{6}$ & 2004 & 728 & TI & $\geq 1,5$ & 63 & 98 & 40 & 98 \\
\hline
\end{tabular}

$\Pi \mathrm{T}=$ troponina $\mathrm{T}, \mathrm{TI}=$ troponina $\mathrm{I}, \mathrm{S}=$ sensibilidade, $\mathrm{E}=$ especificidade, $\mathrm{VPP}=$ valor preditivo positivo, VPN = valor preditivo negativo

${ }^{\star} \mathrm{ECG}=$ presença de eletrocardiograma alterado associado à troponina I elevada. 
Tabela 2 - Evolução dos Indicadores de Necrose Miocárdica após Trauma Torácico Fechado

\begin{tabular}{lccccc}
\hline Tempo (horas) & 0 & 6 & 12 & 18 & 24 \\
\hline Troponina I $(\mu \mathrm{g} / \mathrm{L})$ & 17,1 & 21,1 & 36,2 & 38,3 & 37,6 \\
CKMB massa $(\mathrm{ng} / \mathrm{mL})$ & 87 & 108 & 102 & 61,6 & 27,6 \\
CPK (ug/L) & 3478 & 4516 & 5889 & 6230 & 6562 \\
\hline
\end{tabular}

Valores de referência: Troponina $\mathrm{I}<1 \mu \mathrm{g} / \mathrm{L}$, CKMB massa $<4 \mathrm{ng} / \mathrm{mL}$, CPK $55-170 \mathrm{U} / \mathrm{L}$.

Após 24 horas da admissão, o paciente apresentou piora súbita da função respiratória e necessidade de ventilação mecânica, com quadro clínico compatível com síndrome da angústia respiratória aguda (SARA). Após 48 horas de ventilação mecânica foi possível extubá-lo, sendo mantido em ventilação não-invasiva (BiPap) por mais 24 horas. O paciente obteve alta da UTI para a unidade semi-intensiva com 96 horas de evolução, ventilando espontaneamente e sem complicações cardíacas.

\section{AS TROPONINAS E OS INDICADORES CLÁSSICOS DE LESÃO MIOCÁRDICA}

Ferjani e col. estudaram o papel da troponina $\mathrm{T}$, CKMB atividade e da relação $\mathrm{CKMB}$ atividade/CK total como indicadores de contusão miocárdica em 128 pacientes vítimas de trauma torácico fechado, dos quais 29 (22\%) apresentaram contusão miocárdica ${ }^{7}$. Níveis elevados de troponina $T$ foram significativamente mais freqüentes em pacientes com contusão miocárdica ( $31 \%$ versus $9 \%, p<0,007)$. Não houve diferença estatística significativa no que se refere aos valores da CKMB e da relação CKMB/ CK total nos dois grupos de pacientes estudados. Dos 128 pacientes vítimas de trauma torácico, 95\% (122 pacientes) apresentaram elevação de CK. Neste estudo, a sensibilidade, especificidade, VPP e VPN da Troponina T em predizer contusão miocárdica em trauma torácico fechado foram de 31\%, 91\%, 16\% e $82 \%$, respectivamente; valores muito próximos dos observados com os indicadores tradicionais (CK, CKMB atividade, CKMB atividade/CK total) de lesão miocárdica ${ }^{7}$.

Estudo prospectivo avaliou 88 pacientes politraumatizados, sendo que $43 \%$ apresentavam trauma torácico associado. Embora tenha ocorrido elevação dos níveis séricos de $\mathrm{CKMB}$ atividade, da relação $\mathrm{CKMB}$ atividade/CK total, da CKMB massa e da relação CKMB massa/CK total em $76 \%$ dos pacientes com trauma torácico fechado, não ocorreu, em nenhum desses pacientes, alteração dos níveis séricos de troponina ${ }^{9}$.
Além disso, nenhum dos pacientes com alterações nesses indicadores apresentou sinais clínicos de contusão miocárdica. Dos pacientes politraumatizados sem trauma torácico associado, 57\% apresentavam alteração da CKMB atividade e $20 \%$ apresentaram alteração da CKMB massa na admissão hospitalar. Com 24 horas de evolução, 8\% dos pacientes apresentaram alteração da CKMB atividade e $20 \%$ apresentaram alteração da CKMB massa. Entretanto, nesse grupo de pacientes não ocorreram alterações dos níveis séricos das troponinas I e T.

Esses autores concluíram que as alterações dos níveis de CKMB atividade, CKMB atividade/CK total e CKMB massa/CK total não são úteis para detectar lesão miocárdica em pacientes vítimas de trauma torácico fechado. Os indicadores troponina I e T apresentaram perfil de sensibilidade equivalente e possuem maior precisão para detecção da contusão miocárdica. Esses indicadores auxiliam na seleção dos pacientes com maior risco de eventos adversos e que, portanto, deverão permanecer sob monitorização cardíaca intensiva ${ }^{9}$.

\section{O VALOR PROGNÓSTICO DAS TROPONINAS}

Bertinchant e col. estudaram os valores diagnóstico e prognóstico das troponinas I e T em 94 pacientes consecutivos com trauma torácico fechado ${ }^{5}$. Nesse estudo, 26 pacientes $(27,6 \%)$ apresentaram contusão miocárdica diagnosticada através de alterações ecocardiográficas, eletrocardiográficas ou ambas. A porcentagem de pacientes com níveis plasmáticos elevados de troponinas I e $T$ foi significativamente maior no grupo de pacientes que apresentaram contusão miocárdica $(23 \%$ versus $3 \% ; p=0,01$ e $12 \%$ versus. $0 \%$; $\mathrm{p}=0.03$, respectivamente). A sensibilidade, especificidade, VPP e VPN da Troponina I em predizer contusão miocárdica em trauma torácico fechado foi de $23 \%, 97 \%, 75 \%$ e $77 \%$, respectivamente, enquanto que os valores da troponina $\mathrm{T}$ foram de $12 \%, 100 \%$, $100 \%, 74 \%$.

Não houve mortes em nenhum dos dois grupos de pacientes estudados (com e sem contusão miocárdica) em período médio de seguimento (17 meses). Assim como no estudo de Ferjani ${ }^{7}$, a dosagem seriada de troponina $\mathrm{T}$ apresentou sensibilidade e valor preditivo positivo baixos para o diagnóstico laboratorial de contusão miocárdica. Nesse estudo, não foi possível estabelecer uma relação direta entre os níveis elevados de troponina com a mortalidade e não houve correlação entre a elevação desses indicadores com 
a presença de desfecho clínico precoce e tardio desfavoráveis ${ }^{5}$.

Recentemente, estudo publicado com 333 pacientes avaliou a sensibilidade, a especificidade e os valores preditivos positivo e negativo da troponina I em detectar contusão miocárdica clinicamente significativa, definida como a presença de hipotensão não decorrente de sangramento ou disritmias cardíacas, alterações estruturais pós-traumáticas diagnosticadas pelo ecocardiograma ou índice cardíaco $<2,5 \mathrm{~L} / \mathrm{min} / \mathrm{m}^{210}$.

A presença de contusão miocárdica clinicamente significativa foi constatada em 44 pacientes (13\%). Dos 80 pacientes que apresentaram alterações eletrocardiográficas e elevação de troponina I na admissão hospitalar, 27 (34\%) desenvolveram contusão miocárdica clinicamente significativa. Nenhum paciente com eletrocardiograma e troponina normais na admissão apresentaram essa evolução clínica. Apenas 7\% dos pacientes que apresentaram alteração isolada da troponina I desenvolveram contusão miocárdica com significância clínica ${ }^{10}$.

Nesse estudo, a sensibilidade, a especificidade e os valores preditivos positivo e negativo da troponina I em detectar contusão miocárdica clinicamente significativa foram de $73 \%, 60 \%, 21 \%$ e $94 \%$, respectivamente. A mortalidade foi significativamente maior nos pacientes que apresentaram contusão miocárdica grave $(39 \%$ versus $7 \%, p<0,001)$, assim como o tempo médio de internação hospitalar, duas vezes maior no grupo com contusão grave, em comparação ao grupo sem contusão grave (22 versus 11 dias $p<0,001)$. Nesse mesmo estudo, a associação de troponina I elevada à presença de alterações eletrocardiográficas, produzia melhora significativa da precisão dos métodos em predizer precocemente maior probabilidade do desenvolvimento de contusão miocárdica clinicamente significativa. Esta associação apresentou sensibilidade de $100 \%$, especificidade de $71 \%$, valores preditivos positivo e negativo de $34 \%$ e $100 \%$, respectivamente ${ }^{10}$.

Os autores concluíram que a associação de eletrocardiograma e a troponina I alterados na admissão hospitalar permite detecção precoce dos pacientes que apresentarão contusão miocárdica clinicamente significativa e, assim, com possibilidade de evolução desfavorável. Pacientes com eletrocardiograma e troponina I normais 8 horas após o trauma, apresentariam probabilidade praticamente nula de terem contusão miocárdica significativa e poderiam ter alta hospitalar com segurança ${ }^{10}$.

\section{O PERFIL DE LIBERAÇÃO DAS TROPONINAS}

Edouard e col. desenvolveram estudo prospectivo com 728 pacientes vítimas de trauma torácico fechado em UTI cirúrgica e trauma ${ }^{6}$, com o intuito de determinar a incidência e o padrão de liberação da troponina I e sua correlação com a presença de choque, a contusão miocárdica e a evolução clínica.

A incidência de alteração nos níveis de troponina na população geral estudada foi de $12 \%$ (95\%; Cl 9,6 $-14,4 \%)$. Dos 728 pacientes incluídos no estudo, 35 apresentaram contusão miocárdica significativa, diagnosticada através de critérios eletrocardiográficos (4,8\%; 95\% Cl, 3,4\%-6,6\%). Dos 679 pacientes que sobreviveram após 48 horas do trauma, 54 apresentaram elevação dos níveis de troponina sem que houvesse existência de doença arterial coronariana previa (7\%; 95\% Cl, 5,6\%-9,6\%) ${ }^{6}$.

Lesão cerebral (56\% versus $29 \%$; $p<0.05)$, lesão torácica $(67 \%$ versus $19 \%$; $p<0.05)$, trauma cardíaco fechado $(40 \%$ versus $2 \%$; $p<0.05)$ e choque $(78 \%$ versus $17 \%$; $p<0.05)$ foram mais freqüentes nos pacientes com troponina I elevadas, do que nos pacientes sobreviventes em 48 horas, sem alteração dos níveis de troponina. A mortalidade nos pacientes que sobreviveram após 48 horas do trauma foi semelhante no grupo de pacientes com (15\%; 95\% Cl, 7-27\%) ou sem (12\%; 95\% Cl, 9-14\%) elevação de troponina ${ }^{6}$.

A liberação sustentada (maior que 36 horas) e significativa de troponina I (pico de troponina I $\geq 2 \mu \mathrm{g} / \mathrm{L}$ ) ocorreu em 17 dos 728 pacientes estudados (2,3\% do total; $95 \% \mathrm{Cl}, 1,4 \%-3,7 \%)$, freqüentemente associada ao trauma torácico $(82 \%)$ e a presença de alterações eletrocardiográficas. Anormalidades ecocardiográficas foram encontradas em $53 \%$ desses 17 pacientes (alteração segmentar da contratilidade e derrame pericárdico em 8 e 2 pacientes, respectivamente). A sensibilidade, especificidade, VPP e VPN da troponina I em predizer contusão miocárdica significativa com base nos resultados eletrocardiográficos foi, respectivamente, de $63 \%$, 98\%, 40\% e 98\%. Embora os pacientes tenham sido observados durante 24 meses, não foi possível estabelecer uma relação entre a elevação dos níveis plasmáticos de troponina I com o prognóstico em longo prazo dos pacientes vítimas de contusão miocárdica ${ }^{6}$.

Estudo prospectivo com 187 pacientes politraumatizados, dos quais 63 (34\%) apresentavam contusão miocárdica, o aumento dos níveis séricos de troponina I se correlacionou com aumento da freqüência e da 
gravidade de disritmias $(\alpha=1,791 ; p=0,008)$ e com a presença de depressão temporária da função ventricu$\operatorname{lar}(\alpha=0,523 ; p=0,005)^{8}$. Pacientes que apresentaram troponina I inferior a 1,05 $\mathrm{\mu g} / \mathrm{L}$ na admissão e após um período de observação de 6 horas não apresentaram nenhuma alteração cardíaca durante todo período de seguimento do estudo. Os autores concluíram que pacientes vítimas de trauma torácico fechado com troponina I superior a 1,05 $\mathrm{gg} / \mathrm{L}$ apresentariam risco elevado de arritmias e depressão miocárdica e deveriam permanecer sob observação. Em pacientes assintomáticos que apresentassem troponina I abaixo de 1,05 $\mu \mathrm{g} /$ $L$ após 6 horas de evolução seria possível descartar a presença de contusão miocárdica ${ }^{8}$.

\section{DISCUSSÃO DO CASO CLÍNICO ILUSTRATIVO}

O caso do paciente relatado, vítima de trauma torácico fechado, teve como apresentação clínica uma contusão miocárdica clinicamente significativa, elevação dos níveis plasmáticos de troponina I e disritmia ventricular benigna autolimitada.

De acordo com dados disponíveis na literatura, o esperado é que o paciente apresentasse evolução clinica menos favorável, pela intensa liberação de indicadores de lesão miocárdica, principalmente da troponina $\left.\right|^{6,8,10}$. Entretanto, durante toda a evolução na UTI, não houve sinal de disfunção cardíaca ou a presença de disritmia ventricular maligna. Sua deterioração clínica foi devida à progressão da contusão pulmonar para SARA.

\section{CONCLUSÃO}

Embora exista maior especificidade das troponinas I e T quando comparadas aos indicadores tradicionais, CKMB massa e CPK total, esses dois indicadores apresentam sensibilidade e valor preditivo positivo baixos para diagnosticar contusão miocárdica.

Pacientes vítimas de trauma torácico fechado que apresentam alterações eletrocardiográficas, elevação de troponinas, ou ambas, devem permanecer em observação em UTI, por no mínimo 24 horas, período em que se desenvolve a maioria das complicações decorrentes das contusões miocárdica e pulmonar, secundárias ao trauma. Nesses pacientes, a dosagem seriada de troponina, eletrocardiograma e a realização de um ecocardiograma para avaliação da função ventricular e a presença de possíveis lesões estruturais do coração são fundamentais.

Pacientes vítimas de trauma torácico fechado que estão assintomáticos, hemodinamicamente estáveis, sem alterações eletrocardiográficas e que apresentam troponina I ou T dentro dos limites da normalidade após um período de 12 a 24 horas de observação, apresentam baixa probabilidade de contusão miocárdica, podem ter alta hospitalar com segurança, caso não existam outras indicações para permanecerem internados ${ }^{4}$.

É provável que com a realização de novos estudos seja possível estabelecer uma correlação entre a intensidade de liberação de troponinas, a intensidade e a gravidade da lesão de estruturas adjacentes ao coração, como os pulmões. Espera-se também que novas opções diagnósticas sejam desenvolvidas, contribuindo deste modo com melhora no diagnóstico e tratamento dos pacientes vítimas de trauma torácico fechado.

\section{ABREVIAÇÕES}

$\mathrm{CK}=$ creatinoquinase, $\mathrm{CKMB}=$ creatinoquinase fração $\mathrm{MB}, \mathrm{S}=$ sensibilidade, $\mathrm{E}=$ especificidade, $\mathrm{VPP}=\mathrm{va}-$ lor preditivo positivo e VPN = valor preditivo negativo, $E C G$ = eletrocardiograma.

\section{REFERÊNCIAS}

01. Ministério da Saúde/SE/Datasus - Sistema de Informações Hospitalares do SUS-SIH/SUS. Ministério da Saúde/SE/Datasus, 2005.

02. Elie MC - Blunt cardiac injury. Mt Sinai J Med, 2006;73:542-552.

03. Kaye P, O'Sullivan I - Myocardial contusion: emergency investigation and diagnosis. Emerg Med J, 2002;19:8-10.

04. Sybrandy KC, Cramer MJ, Burgersdijk C - Diagnosing cardiac contusion: old wisdom and new insights. Heart, 2003;89:485-489.

05. Bertinchant JP, Polge A, Mohty D et al - Evaluation of incidence, clinical significance, and prognostic value of circulating cardiac troponin I and T elevation in hemodynamically stable patients with suspected myocardial contusion after blunt chest trauma. J Trauma, 2000;48:924-931.

06. Edouard AR, Felten ML, Hebert $\mathrm{JL}$ et al - Incidence and significance of cardiac troponin I release in severe trauma patients. Anesthesiology, 2004;101:1262-1268.

07. Ferjani M, Droc G, Dreux S et al - Circulating cardiac troponin $\mathrm{T}$ in myocardial contusion. Chest, 1997;111:427-433.

08. Rajan GP, Zellweger R - Cardiac troponin I as a predictor of arrhythmia and ventricular dysfunction in trauma patients with myocardial contusion. J Trauma, 2004;57:801-808.

09. Swaanenburg JC, Klaase JM, DeJongste MJ et al - Troponin I, troponin T, CKMB-activity and CKMB-mass as markers for the detection of myocardial contusion in patients who experienced blunt trauma. Clin Chim Acta, 1998;272:171-181.

10. Velmahos GC, Karaiskakis M, Salim A et al - Normal electrocardiography and serum troponin I levels preclude the presence of clinically significant blunt cardiac injury. J Trauma, 2003;54:45-51 